\title{
Endoscopic sphincterotomy and risk of cholangio- carcinoma: a population-based cohort study in Finland and Sweden
}

Authors

Institutions
Cecilia Strömberg ${ }^{1,2}$, Camilla Böckelman ${ }^{3}$, Huan Song ${ }^{4}$, Weimin Ye ${ }^{4}$, Eero Pukkala ${ }^{5,6}$, Caj Haglund7 ${ }^{7}$ Magnus Nilsson ${ }^{1,2}$

Institutions are listed at the end of article. submitted 30. March 2016 accepted after revision 29. July 2016

\section{Bibliography}

DOI http://dx.doi.org/

10.1055/s-0042-114982

Published online: 14.9.2016

Endoscopy International Open 2016; 04: E1096-E1100

(c) Georg Thieme Verlag KG

Stuttgart · New York

E-ISSN 2196-9736

\section{Corresponding author}

Cecilia Strömberg, MD, PhD

Division of Surgery

Centre for Digestive

Diseases K53

Karolinska University Hospital

Hälsov 141

14186 Huddinge

Sweden

Fax: +46-8-58586910

cecilia.stromberg@karolinska.se

\section{License terms}

(ब)(1) $\Theta \circledast$
Background and study aims: Elevated long-term risk of cholangiocarcinoma is reported after endoscopic sphincterotomy (ES), but in a previous study we found a trend towards a decreased risk. The aim of this study was to evaluate the association in a larger cohort with a longer follow-up. Patients and methods: Data concerning all patients having had an inpatient endoscopic retrograde cholangiopancreatography (ERCP) were collected from the hospital discharge registries of Finland and Sweden. Incident cases of malignancy were identified through linkage to the nationwide Cancer Registries. Patients with a diagnosis of malignancy, before or within 2 years of the ERCP, were excluded. The cohorts were followed until a diagnosis of malignancy, death or emigration, or end of follow-up (end of 2010). The relative risk of malignancy was calculated as standardized incidence ratio (SIR) compared with the

\section{Introduction}

Endoscopic retrograde cholangiopancreatography (ERCP) with endoscopic sphincterotomy (ES) was first introduced in 1974 in Germany and Japan $[1,2]$ and a few years later in the Nordic countries, as an alternative to open surgery with choledochotomy in the treatment of common bile duct stones. It was mainly used in elderly patients or patients with severe comorbidity, in whom mortality after ERCP was reduced compared to open surgery [3], whereas young and healthy patients were still predominantly treated with open surgical procedures. Since the introduction of laparoscopic cholecystectomy, ERCP with ES, with or without cholecystectomy, has become the most common procedure in the treatment of common bile duct stones worldwide. A consequence of this alteration in therapeutic regime is that an increased number of young patients, with a long life expectancy, currently undergo ES. general population, inherently adjusting for age, gender, and calendar year of follow-up.

Results: A total of 69925 patients undergoing ERCP from 1976 through 2008 were included in the pooled cohort. ES was performed in 40193 subjects. The risk of malignancy was elevated in the total cohort (SIR $=2.3$; 95\% confidence interval [CI] $2.1-2.5$ ) irrespective of whether ES was performed or not. The SIRs diminished with duration of follow-up.

Conclusions: We found an elevated risk of malignancy both in the bile ducts alone and in the bile ducts, liver or pancreas together, after ERCP. The risk was the same, regardless of whether ES had been performed or not, so ES was unlikely to be the cause, and a common carcinogenic exposure previous to the ERCP procedure, possibly ductal gallstone disease, was more likely.

Reports demonstrate the long-term adverse effects of ES.Interruption of the sphincter of Oddi has been reported to cause overgrowth of bacteria [4] in the common bile duct resulting in cholangitis and recurrent stone formation [5]. Studies also show an increased incidence of cholangiocarcinoma after ES, presumably due to the bacterial overgrowth and chronic inflammation in the bile ducts $[5,6]$. This is in agreement with other reports concerning the development of malignancies after open bilioenteric anastomoses [7,8], and transduodenal sphincterotomy [9]. These data prompted us to hypothesize that ES increases the long-term risk of cholangiocarcinoma, which we were subsequently able to test in a Swedish nationwide population-based study of all 27708 patients registered for an ERCP for benign disease in the Swedish inpatient registry, since the introduction of ERCP in the 1970s until 2005 [10]. Our results showed that, among the 12629 patients who had undergone ES, the risk of cancer in the bile ducts was not increased compared to the 


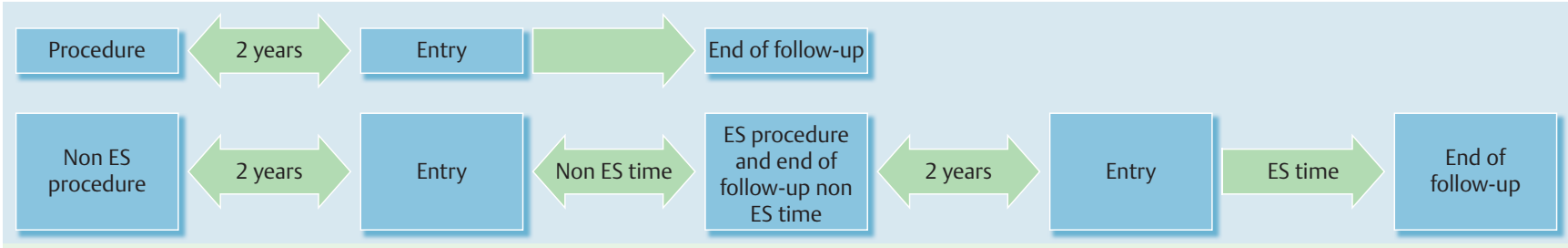

Fig. 1 Calculation of follow-up time in patients with one or several procedures. End of follow-up was 31 December 2010, a diagnosis of malignancy, death, emigration or second procedure.

non-ES group, but on the other hand, that there was a tendency toward a decreased long-term risk of bile duct cancer in the ES group. However, due to the small number of patients followed for more than 10 years, our data did not have the statistical power to address this question. In this study, we also found a decreased risk of malignancy in patients who had had a cholecystectomy. The primary aim of this population-based cohort study of all patients registered for an ERCP in the inpatient registries in Finland and Sweden was to study the risk of malignancy in the bile ducts in a larger cohort and with a longer follow-up. A secondary aim was to study the relationship between severe common bile duct stone exposure, which is a valid assumption for the entire ERCP cohort irrespective of ES, and malignancy in the biliary tract. Because of expected extensive misclassification between extrahepatic bile duct cancers and pancreatic cancer, as well as between intrahepatic bile duct cancers and liver cancer, both bile duct cancer alone as well as bile duct cancer together with pancreatic and liver cancers, were used as outcome parameters.

\section{Materials and methods \\ $\nabla$}

\section{Registries, cohort and follow-up}

We used data from the Finnish and Swedish hospital discharge registers, in which discharge diagnoses and surgical procedures are computerized for each hospitalization. Unique national registration numbers for each inhabitant are in use in both Finland and Sweden, and we used them for identification. The coverage of the registries is nearly $100 \%$ from 1986 and onwards [11,12]. The hospital discharge registries were introduced 1986 in Finland and 1965 in Sweden, and the first ERCP procedure was registered in 1976. We identified patients from 1986 to 2010 in Finland and 1976 to 2010 in Sweden with at least one in-hospital episode with a discharge procedure code for ERCP or endoscopic sphincterotomy. In the ERCP group, patients with a code of ERCP with or without biopsy and cholangioscopy were included while the ES group included patients with codes of sphincterotomy, extraction of stones, insertion of stent or nasobiliary drainage (International Classification of Operations and Major Procedures, codes 9014, UJK02, UJK05, UJK12, UJK15 for ERCP or 5388, 5394, JKE 02, JKE 12, JKE 15, JKE 18, JKE 25, JKE 98 for ES or procedures for which $E S$ is normally a prerequisite). Using the national registration number, the patients identified with the procedure codes listed above were linked to each country's nationwide cancer registry, population registry, and migration registry for cancer outcome ascertainment and censoring due to death and emigration, respectively. A detailed description of the methods used has been described elsewhere [13].

For further analyses, the total ERCP cohort for each country was divided into two subgroups: (1) patients having at least one procedure code registration for ES or any other endoscopic biliary procedures for which an ES is normally a prerequisite (International Classification of Operations and Major Procedures, codes 5388, 5394, JKE 02, JKE 12, JKE 15, JKE 18, JKE 25, JKE 98), and (2) patients in the cohort without any procedure code registration for ES or any other endoscopic biliary procedure implying ES. Those patients who had a diagnosis of malignant tumor in the bile ducts, liver or pancreas (ICD-O-3 C22-25 in the Finnish data and ICD7 155-157 in the Swedish data or corresponding codes in the later classifications) at the time of the procedure or within 2 years after it, were excluded from further analyses to avoid selection bias, since the registered ERCP in these cases may have been performed because of the tumor or due to symptoms caused by a tumor that was still undiagnosed. Considering the poor prognosis of malignancies in the biliary tract, liver and pancreas, it is highly unlikely that a tumor causing symptoms would be diagnosed more than 2 years later. The cohorts were then followed from entry ( 2 years after the procedure) until diagnosis of an outcome malignancy (primary malignant tumors in the liver, bile ducts including ampullary region and pancreas, but excluding gallbladder malignancy, ICD7 codes: 155 and 157, but excluding 1551 and ICD10 codes C22.1, C24.0, C24.1, C24.8, and $\mathrm{C25}$ ), death, emigration or end of follow-up (31 December 2010), whichever occurred first.

The study was approved by the National Institute for Health and Welfare in Finland and the Regional Research Ethics Committee of Stockholm and Helsinki.

\section{Statistical analyses}

Several patients had ERCP or ES procedures registered more than once. For each patient, every first-time procedure was regarded as the index procedure for the corresponding cancer analyses. If the first-time procedure included or implied ES, the patient's person-time was only included in the ES subgroup. If a patient's first procedure was non-ES or ES-implying followed by a subsequent procedure implying ES, this patient had two index procedures: one without ES, with person-time counted from 2 years after the procedure until the subsequent ES procedure (i.e. censored at the time of the ES procedure); and another one after which person-time was counted in the ES subgroup from 2 years after the ES procedure. However, for the whole ERCP group, person-time was counted from 2 years after entry until the occurrence of cancer, death, emigration or end of follow-up, irrespective of ES procedure ( Fig. 1 ). Thus the total person-time in the whole ERCP cohort was more than the sum of the person-time experienced by the two subgroups.

The standardized incidence ratio (SIR), the ratio of the observed to the expected number of malignancies, was used to calculate relative risk. The background cancer incidence rates for the general Swedish and Finish populations were provided by the national cancer registries in the corresponding country, which was the same as the source for linkage to identify cancer cases 
during follow-up. The expected number of cancers was calculated by using the sex, calendar, age, and country-specific incidence rates. The standardized incidence ratios are inherently adjusted for confounding by age at follow-up, gender, and calendar year of follow-up.

To calculate the pooled SIR, we first combined the number of observed cancers, $\sum \mathrm{O}$, by summing data from the two countries, and the combined number of expected cancers, $\sum \mathrm{E}$, was determined in a similar way. The pooled SIR was calculated as $\sum \mathrm{O} / \sum \mathrm{E}$. The 95\% confidence intervals (CIs) for the pooled SIR were determined by assuming a Poisson distribution of the observed numbers of cancer. Results using the weighted meta-analysis were essentially identical with those using the pooled method, and tests for heterogeneity using the chi-squared test as well as $\mathrm{I}^{2}$ statistics demonstrated that the inconsistencies across the two studies were minor $\left(X^{2}=0.19, \mathrm{df}=1, P=0.66 ; \mathrm{I}^{2}=0 \%\right.$ ). Therefore, we just present the data from the pooled analyses [14-16]. Further analyses were stratified by duration of follow-up $(2-4,5-9, \geq 10$ years). The excess absolute risk, i.e. difference between observed and expected number of cases divided by person-years $\left(\sum(\mathrm{O}-\mathrm{E})\right.$ Eperson-years $\times 100000$ ), was also calculated.

A two-sided $P$ value less than 0.05 was considered to be statistically significant. All analyses were conducted with SAS statistical software, version 9.4 (Cary, North Carolina, United States).

\section{Results \\ $\nabla$}

The final cohorts of patients having undergone ERCP, for diagnostics or therapy of non-malignant diseases, included 16575 patients in Finland and 53350 patients in Sweden contributing to a total of 506998 person-years of follow-up. Table 1 shows the steps for selection of patients from the total cohorts including all ERCP procedures to the eligible final benign disease ERCP cohorts. The sums of the ES and non-ES groups are larger than the all ERCP groups, because a number of patients had an ERCP without ES before a subsequent ERCP with ES. Of these, 784 did not have a diagnosis of malignancy at the time of the first or second index procedures or within 2 years thereafter, and were thus counted in both subgroups.

The general characteristics of the cohorts and the number of observed malignancies 2 years or more after the index procedure are included in Table 2 . The mean age at entry, 2 years after the procedure, was 66 years in Sweden and 64 years in Finland. There was a predominance of women in both countries. The mean follow-up time was 7.3 years in the all ERCP cohort, but was shorter among patients who had undergone an ES. Bile duct malignancy was infrequent and despite the large size of the cohort, the number of bile duct malignancies in the studied population was low. The risk of developing malignancy in the bile ducts, liver, or pancreas, explicitly excluding gallbladder malignancy, was increased in the all ERCP cohorts, the ES cohorts, and the ERCP without ES cohorts in both Finland and Sweden (data not shown). In the pooled all ERCP cohort, the risk was more than twofold compared to the general national populations (SIR 2.3; 95\%CI 2.1-2.5). Likewise, it was increased in the pooled ES subgroup (SIR 2.2; $95 \% \mathrm{CI} 2.0-2.5$ ) and almost the same in the non-ES subgroup (SIR 2.1; 95\%CI 1.9-2.3) ( Table 3). The relative risk of bile duct malignancy alone, was increased nearly four times (SIR 3.9; 95\% CI 3.3 - 4.5) in the pooled all ERCP cohort, and tended to be higher in the ES cohort (SIR 4.3; 95\%CI 3.5 - 5.5) than in the cohort without ES (SIR 2.7; 95\%CI 2.0-3.5) ( Table 3).
Table 1 Size of cohorts of patients having undergone ERCP, ERCP with ES and ERCP without ES in Finland and Sweden.

\begin{tabular}{|llll|}
\hline & All ERCP & ES $^{1}$ & $\begin{array}{l}\text { ERCP without } \\
\text { ES }^{1}\end{array}$ \\
\hline Total & 117416 & 72331 & 49896 \\
\hline $\begin{array}{l}\text { Operation date before entry } \\
\text { (1976-01-01 for Sweden }\end{array}$ & 14 & 2 & 13 \\
\hline $\begin{array}{l}\text { and 1986-01-01 for Finland) } \\
\text { Error in registration }\end{array}$ & 1645 & 1121 & 580 \\
\hline $\begin{array}{l}\text { Cancer diagnosis } \\
\text { Fofore entry }\end{array}$ & 8583 & 5380 & 3443 \\
\hline $\begin{array}{l}\text { Follow-up less than 2 years } \\
\text { Eligible }\end{array}$ & 37249 & 25635 & 15344 \\
\hline
\end{tabular}

ERCP, endoscopic retrograde cholangiopancreatography; ES, endoscopic sphincterotomy.

${ }^{1}$ The sum of the ES group and ERCP without ES group was more than the whole ERCP cohort, since some patients contributed person-time to both groups.

2 Diagnosis of malignant tumor in the bile ducts, liver or pancreas.

Table 2 Selected characteristics and number of patients developing malignancy more than 2 years after ERCP with or without ES in Finland and Sweden.

\begin{tabular}{|c|c|c|c|}
\hline & $\begin{array}{l}\text { All ERCP } \\
(n=69925)\end{array}$ & $\begin{array}{l}\text { ES } \\
(n=40193)\end{array}$ & $\begin{array}{l}\text { ERCP without } \\
\text { ES } \\
(n=30516)\end{array}$ \\
\hline $\begin{array}{l}\text { Mean }( \pm S D) \text { age at entry, } \\
\text { years }\end{array}$ & $65.4 \pm 17.7$ & $67.4 \pm 17.7$ & $62.7 \pm 17.8$ \\
\hline Sex (\% male) & 41.8 & 40.8 & 43.2 \\
\hline Mean of follow-up, years & 7.3 & 5.3 & 9.4 \\
\hline Person-years at risk & 506998 & 162682 & 238561 \\
\hline $\begin{array}{l}\text { Malignancy of bile ducts, } \\
\text { liver, or pancreas exclud- } \\
\text { ing gallbladder, cases }\end{array}$ & 670 & 289 & 330 \\
\hline $\begin{array}{l}\text { Malignancy of bile ducts } \\
\text { (ICD-7 155, excluding } \\
1550 \text { and 1551) }\end{array}$ & 150 & 79 & 55 \\
\hline $\begin{array}{l}\text { Malignancy of pancreas } \\
\text { (ICD-7 157) }\end{array}$ & 309 & 139 & 146 \\
\hline
\end{tabular}

ERCP, endoscopic retrograde cholangiopancreatography; ES, endoscopic sphincterotomy.

Table 3 Pooled SIRs and their $95 \%$ confidence intervals (CI) for diagnosis of malignancies 2 years or more after ERCP with or without ES on a benign indication.

\begin{tabular}{|c|c|c|c|}
\hline & $\begin{array}{l}\text { All ERCP } \\
(n=69925)\end{array}$ & $\begin{array}{l}\text { ES } \\
(n=40193)\end{array}$ & $\begin{array}{l}\text { ERCP without } \\
\text { ES } \\
(n=30516)\end{array}$ \\
\hline & SIR (95\%CI) & SIR (95\%CI) & SIR (95\%CI) \\
\hline $\begin{array}{l}\text { Malignancy of the bile } \\
\text { ducts, liver, or pancreas } \\
\text { excluding gallbladder }\end{array}$ & $\begin{array}{l}2.3 \\
(2.1-2.5)\end{array}$ & $\begin{array}{l}2.2 \\
(2.0-2.5)\end{array}$ & $\begin{array}{l}2.1 \\
(1.9-2.3)\end{array}$ \\
\hline $\begin{array}{l}\text { Malignancy of the } \\
\text { bile ducts }\end{array}$ & $\begin{array}{l}3.9 \\
(3.3-4.5)\end{array}$ & $\begin{array}{l}4.4 \\
(3.5-5.5)\end{array}$ & $\begin{array}{l}2.7 \\
(2.0-3.5)\end{array}$ \\
\hline $\begin{array}{l}\text { Malignancy of the } \\
\text { pancreas }\end{array}$ & $\begin{array}{l}1.8 \\
(1.6-2.0)\end{array}$ & $\begin{array}{l}1.7 \\
(1.5-2.1)\end{array}$ & $\begin{array}{l}1.5 \\
(1.3-1.8)\end{array}$ \\
\hline
\end{tabular}

ERCP, endoscopic retrograde cholangiopancreatography; ES, endoscopic sphincterotomy; SIR, standardized incidence ratio.

The SIR of malignancy of the bile ducts, liver, or pancreas was much elevated in the first two years after the procedure at 69.2 (95\%CI 66.8-71.6), but then decreased gradually with increasing follow-up duration. In the pooled all ERCP cohort in the period between 2 and 5 years after the index ERCP, the SIR was $3.0(95 \% \mathrm{CI}$ $2.7-3.5)$ and decreased to 1.9 (95\%CI $1.6-2.2)$ for 10 years and 
Table 4 Pooled SIRs with their 95\% confidence intervals (CI) for developing malignancy in the bile ducts, liver, or pancreas after ERCP, with or without ES, by duration.

\begin{tabular}{|c|c|c|c|c|c|c|c|c|c|c|c|c|}
\hline \multirow[b]{2}{*}{$\begin{array}{l}\text { Years after } \\
\text { procedure }\end{array}$} & \multirow[b]{2}{*}{ E } & \multicolumn{4}{|c|}{ All ERCP $(n=69925)$} & \multicolumn{4}{|c|}{$E S(n=40193)$} & \multicolumn{3}{|c|}{ ERCP without ES $(n=30516)$} \\
\hline & & 0 & SIR (95\%CI) & $\begin{array}{l}\text { Excess ab- } \\
\text { solute risk }{ }^{1}\end{array}$ & E & 0 & SIR $(95 \% \mathrm{Cl})$ & $\begin{array}{l}\text { Excess ab- } \\
\text { solute risk }{ }^{1}\end{array}$ & E & 0 & $\operatorname{SIR}(95 \% \mathrm{CI})$ & $\begin{array}{l}\text { Excess ab- } \\
\text { solute risk }{ }^{1}\end{array}$ \\
\hline $2-4$ & 75.7 & 230 & $3.0(2.7-3.5)$ & 122.8 & 42.9 & 131 & $3.1(2.6-3.6)$ & 125.9 & 33.4 & 97 & $2.9(2.4-3.5)$ & 112.2 \\
\hline $5-9$ & 122.6 & 266 & $2.2(1.9-2.4)$ & 68.1 & 61.0 & 123 & $2.0(1.7-2.4)$ & 61.2 & 61.4 & 124 & $2.0(1.7-2.4)$ & 57.4 \\
\hline$\geq 10$ & 92.8 & 174 & $1.9(1.6-2.2)$ & 47.6 & 26.2 & 35 & $1.3(0.9-1.9)$ & 19.5 & 64.6 & 109 & $1.7(1.4-2.0)$ & 36.4 \\
\hline
\end{tabular}

ERCP, endoscopic retrograde cholangiopancreatography; ES, endoscopic sphincterotomy; SIR, standardized incidence ratio.

${ }^{1}$ Calculated from $(\mathrm{O}-\mathrm{E}) /$ person-years $\times 100000$.

Table 5 Pooled SIRs with their $95 \%$ confidence intervals $(\mathrm{Cl})$ for developing malignancy in the bile ducts after ERCP, with or without ES, by duration.

\begin{tabular}{|c|c|c|c|c|c|c|c|c|c|c|c|c|}
\hline \multirow[b]{2}{*}{$\begin{array}{l}\text { Years after } \\
\text { procedure }\end{array}$} & \multirow[b]{2}{*}{ E } & \multicolumn{4}{|c|}{ All ERCP $(n=69925)$} & \multicolumn{4}{|c|}{$\operatorname{ES}(n=40193)$} & \multicolumn{3}{|c|}{ ERCP without ES $(n=30516)$} \\
\hline & & 0 & SIR (95\%Cl) & $\begin{array}{l}\text { Excess ab- } \\
\text { solute risk }^{1}\end{array}$ & E & 0 & SIR (95\%Cl) & $\begin{array}{l}\text { Excess ab- } \\
\text { solute risk }\end{array}$ & E & 0 & $\operatorname{SIR}(95 \% \mathrm{Cl})$ & $\begin{array}{l}\text { Excess ab- } \\
\text { solute risk }\end{array}$ \\
\hline $2-4$ & 10.1 & 64 & $6.4(4.9-8.1)$ & 42.9 & 5.9 & 40 & $6.8(4.8-9.2)$ & 48.8 & 4.2 & 25 & $5.9(3.8-8.7)$ & 36.6 \\
\hline $5-9$ & 16.3 & 56 & $3.4(2.6-4.4)$ & 18.8 & 8.4 & 32 & $3.8(2.6-5.3)$ & 23.3 & 7.9 & 17 & $2.1(1.3-3.4)$ & 8.3 \\
\hline$\geq 10$ & 12.3 & 30 & $2.4(1.6-3.5)$ & 10.4 & 3.6 & 7 & $1.9(0.8-4.0)$ & 7.5 & 8.4 & 13 & $1.5(0.8-2.6)$ & 3.8 \\
\hline
\end{tabular}

ERCP, endoscopic retrograde cholangiopancreatography; ES, endoscopic sphincterotomy; SIR, standardized incidence ratio.

${ }^{1}$ Calculated from $(\mathrm{O}-\mathrm{E}) /$ person-years $\times 100000$.

more after the index ERCP. The excess absolute risks were similarly decreasing with follow-up duration ( $\bullet$ Table 4$)$. The analyses stratified by ES showed a similar pattern, with decreasing pointestimates for relative risks and absolute risks with longer followup. The risk of developing cancer in the bile ducts after ERCP, with or without ES also decreased with length of follow-up, but since cholangiocarcinoma is a rare tumor, the confidence intervals are wide despite the large size of the cohort ( $\bullet$ Table 5 ).

\section{Discussion}

\section{$\nabla$}

This large, population-based cohort study was designed primarily to investigate the risk of developing malignancy in the bile ducts after ES in a large cohort with a long follow-up, a concern raised in other publications $[5,17,18]$. We demonstrate here a fourfold significantly increased risk of malignancy in the bile ducts alone after ERCP, and not significantly higher after ES. Among patients who had undergone ERCP, with or without ES, we found a twofold increased risk of malignancy in the bile ducts, liver, or pancreas. In both the ES and non-ES groups, the absolute excess risks as well as the SIRs of developing a malignancy in the bile ducts, liver, and pancreas together, decreased with increasing length of follow-up.

The strengths of our study include the population-based cohort design with follow-up of a vast majority of patients having undergone ERCP procedures in Finland and Sweden during the study-period. Another strength is the close to complete followup of the patients in the registries used in this study, avoiding differential misclassification and thus ensuring high internal validity. The consistent results in two separate countries could add to the reproducibility. Furthermore, the large size of the cohort provided adequate statistical power, which enabled us to analyze the relative risks of the studied malignancies in the bile ducts, liver, and pancreas after stratification for ES.

A limitation of this study may be that cancer of the bile ducts is a rare malignancy with a low expected incidence even in a large pooled cohort, limiting the subgroup analyses of cancer risk by follow-up duration. The mean follow-up time was shorter in the ES subgroup than in the ERCP without ES group in both the Finnish and Swedish cohorts, as a result of the fact that ES was not performed at the beginning of the period. However, the size of the pooled cohort gives an opportunity to interpret data with a better precision than in previous studies. The risk of misclassification between intrahepatic cholangiocarcinoma and liver cancer, as well as between extrahepatic cholangiocarcinoma and pancreatic cancer must be acknowledged. Therefore, both bile duct malignancy alone as well as bile duct malignancy together with liver and pancreatic malignancies, were used as outcomes in spite of the fact that our hypothesis primarily concerned cholangiocarcinoma of the intrahepatic and extrahepatic bile ducts. The influence of this type of misclassification is predictable and thus tolerable given the use of both outcomes described above.

This study demonstrates an approximately fourfold increase in the risk of malignancy in the bile ducts alone and a twofold increase in the bile ducts, liver and pancreas together, in a large cohort of patients followed up after ES. This increased risk is not likely to be caused by the ES procedure in itself. If that were the case, one would expect to identify a non-elevated risk of tumors at the beginning of the follow-up period and then a gradual increase over time. On the contrary, in this cohort, both in the ES subgroup and in the non-ES subgroup, the relative risk of malignancy was greatest at the beginning of follow-up, i.e. between 2 and 5 years after the index procedure.

In a study of the long-term consequences of ES by Tanaka et al. [5], 410 patients were followed on average for 10 years after ES, and carcinoma in the biliary tract was found in eight patients, three of them late, giving a marked elevated risk of malignancy both in the short and long term, a finding that was attributed to the ES. The result of this study differs from ours by showing a much higher risk of development of carcinoma in the biliary tract, but is based on a much smaller cohort with a higher risk of random error. Fujimoto et al. [17] reported a long-term risk of malignancy in the biliary system, but predominantly gallbladder can- 
cer, of $3.1 \%$ after ES, a rate they concluded to be comparable with the incidence of gallbladder cancer found in patients with gallstone disease.

On the other hand, in a previously published population-based study of 992 patients who underwent ES at six different hospitals, Karlsson et al. found no increase in the risk of cancer in the liver, bile ducts, or pancreas at follow-up 1 year or more after the procedure [19]. This study, also performed in Sweden, is based on a regional subgroup of our own study cohort.

In a large population-based Danish study, Mortensen et al. [20] compared more than 20000 patients undergoing ERCP with or without ES and found a high incidence of cholangiocarcinoma in both groups in the first year after the procedure but a decreasing incidence with length of follow-up, the same result as in the present study, and concluded a lack of causal association between ES and cholangiocarcinoma.

Our study shows an increased risk of malignancy after ERCP but since the risk diminishes with length of follow-up, it is likely to be caused by some common exposure before the ERCP procedure, a conclusion well in line with the Danish findings and the result of our previous study. By far the most likely candidate is gallstone disease, in particular, ductal gallstone disease. In our previous study [10], based on a subgroup of the present cohort, we found that patients who had ever had a cholecystectomy had a lower risk of malignancy, irrespective of ES, a finding that could support this theory. We also calculated the risk of lung cancer as an estimate for potential bias of tobacco smoking, a known risk factor for pancreatic carcinoma, and found no elevated risk compared to the general population.

All patients with a diagnosis of malignancy in the biliary tract, liver, or pancreas at the time of the index procedure, or within 2 years after it, were excluded from the cohort. Patients having uncharacteristic abdominal discomfort could have been subject to ERCP on the assumption of gallstone disease when the symptoms were instead caused by an undiagnosed malignancy explaining the extremely elevated SIRs. We also excluded all patients who were diagnosed with gallbladder malignancy since gallbladder carcinoma is known to be strongly associated with gallstone disease [21], a condition likely to be very common among patients having had an ERCP without a malignant diagnosis.

The risk of malignancy is highly elevated 2 years after ERCP. It is unlikely to be explained by a preexisting undiagnosed cancer at the time of the index procedure, considering the poor prognosis of symptom-generating malignancies in the area. One possible mechanism by which ductal gallstones could cause the high risk of malignancy observed during this part of the follow-up, could be that they may have started a slow, initially purely inflammatory but subsequently carcinogenic, process before they were removed and that the process continued after removal of the stones.

In conclusion, this study shows an elevated risk of malignancy both in the bile ducts alone and in the bile ducts, liver or pancreas together, after ERCP. The risk is not influenced by including ES so ES or any other part of the procedure is unlikely to be the cause. The elevated risk of malignancy is more likely to be caused by some other carcinogenic exposure prior to the ERCP procedure, possibly ductal gallstone disease.

Competing interests: No conflicts of interest exist.

\section{Institutions}

${ }^{1}$ Division of Surgery, Department of Clinical Science, Intervention and Technology, Karolinska Institutet, Stockholm, Sweden

${ }^{2}$ Centre for Digestive Diseases, Karolinska University Hospital, Stockholm, Sweden

3 Department of Surgery, Vaasa Central Hospital, Vaasa, Finland

${ }^{4}$ Department of Medical Epidemiology and Biostatistics, Karolinska Institutet,

Stockholm, Sweden

${ }^{5}$ Finnish Cancer Registry, Institute for Statistical and Epidemiological Cancer

Research, Helsinki, Finland

6 School of Health Sciences, University of Tampere, Tampere, Finland

7 Department of Surgery, University of Helsinki and Helsinki University

Hospital, Finland

\section{References}

1 Classen M, Demling L. [Endoscopic sphincterotomy of the papilla of vater and extraction of stones from the choledochal duct (author's transl)]. Dtsch Med Wochenschr 1974; 99: 496-497

2 Kawai K, Akasaka Y, Murakami K et al. Endoscopic sphincterotomy of the ampulla of Vater. Gastrointest Endosc 1974; 20: 148 -151

3 Davidson BR, Neoptolemos JP, Carr-Locke DL. Endoscopic sphincterotomy for common bile duct calculi in patients with gall bladder in situ considered unfit for surgery. Gut 1988; 29: 114-120

4 Gregg JA, De Girolami P, Carr-Locke DL. Effects of sphincteroplasty and endoscopic sphincterotomy on the bacteriologic characteristics of the common bile duct. Am J Surg 1985; 149: 668-671

5 Tanaka $M$, Takahata $S$, Konomi $H$ et al. Long-term consequence of endoscopic sphincterotomy for bile duct stones. Gastrointest Endosc 1998; 48: 465 - 469

6 Tranter SE, Thompson MH. Comparison of endoscopic sphincterotomy and laparoscopic exploration of the common bile duct. Br J Surg 2002; 89: 1495 - 1504

7 Haratake J, Horie A, Takeda N. [Cancer of the intra-pancreatic common bile duct observed 9 years after choledochojejunostomy]. Gan no rinsho 1983; 29: $1367-1370$

8 Shields HM. Occurrence of an adenocarcinoma at the choledochoenteric anastomosis 14 years after pancreatoduodenectomy for benign disease. Gastroenterology 1977; 72: 322 - 324

9 Tocchi A, Mazzoni G, Liotta G et al. Late development of bile duct cancer in patients who had biliary-enteric drainage for benign disease: a follow-up study of more than 1,000 patients. Ann Surg 2001; 234: 210 214

10 Stromberg C, Luo J, Enochsson $L$ et al. Endoscopic sphincterotomy and risk of malignancy in the bile ducts, liver, and pancreas. Clin Gastroenterol Hepatol 2008; 6: 1049-1053

11 Fall $K$, Ye $W$, Nyren $O$. Risk for gastric cancer after cholecystectomy. Am J Gastroenterol 2007; 102: 1180 - 1184

12 Sund R. Quality of the Finnish Hospital Discharge Register: a systematic review. Scand J Publ Health 2012; 40: 505-515

13 Nyren O, McLaughlin JK, Gridley G et al. Cancer risk after hip replacement with metal implants: a population-based cohort study in Sweden. J Natl Cancer Inst 1995; 87: 28-33

14 Lens MB, Newton-Bishop JA. An association between cutaneous melanoma and non-Hodgkin's lymphoma: pooled analysis of published data with a review. Ann Oncol 2005; 16: 460-465

15 Lipworth L, Tarone RE, Friis $S$ et al. Cancer among Scandinavian women with cosmetic breast implants: a pooled long-term follow-up study. Int J Cancer 2009; 124: 490-493

16 Scheurer ME, Etzel CJ, Liu $M$ et al. Familial aggregation of glioma: a pooled analysis. Am J Epidemiol 2010; 172: 1099-1107

17 Fujimoto T, Tsuyuguchi T, Sakai Y et al. Long-term outcome of endoscopic papillotomy for choledocholithiasis with cholecystolithiasis. Dig Endosc 2010; 22: 95 - 100

18 Pereira-Lima JC, Jakobs $R$, Winter $U H$ et al. Long-term results (7 to 10 years) of endoscopic papillotomy for choledocholithiasis. Multivariate analysis of prognostic factors for the recurrence of biliary symptoms. Gastrointest Endosc 1998; 48: 457-464

19 Karlson BM, Ekbom A, Arvidsson D et al. Population-based study of cancer risk and relative survival following sphincterotomy for stones in the common bile duct. Br J Surg 1997; 84: 1235-1238

20 Mortensen FV, Jepsen P, Tarone RE et al. Endoscopic sphincterotomy and long-term risk of cholangiocarcinoma: a population-based follow-up study. J Natl Cancer Inst 2008; 100: 745-750

21 Tazuma S, Kajiyama G. Carcinogenesis of malignant lesions of the gall bladder. The impact of chronic inflammation and gallstones. Langenbecks Arch Surg 2001; 386: 224-229 